\title{
REVIEW ON A NEW GENERATION WIRELESS MOBILE NETWORK -
}

\author{
5G \\ Rita C. Nilawar ${ }^{1}$, D.M. Bhalerao ${ }^{2}$ \\ ${ }^{1}$ Student, Department of E\&TC, Sinhgad college of engineering, Pune -41, Maharashtra, India \\ ${ }^{2}$ Professor, Department of E\&TC, Sinhgad college of engineering, Pune -41, Maharashtra, India
}

\begin{abstract}
A widespread study related to $5 G$ of mobile communication is the objective of this paper. $5 G$ researches are associated to the improvement of $W W W W$ (i.e. world wise wireless web.) DAWN (i.e. dynamic adhoc wireless network) and existent mobile wireless communication The most significant technologies for 5G are Wireless Local Area Network (WLAN) and Wireless Metropolian Area Network (WMAN), Wireless Personal Area Network (WPAN) in digital communication. This paper mainly contributes to the key provisions of $5 G$ technology. In $5^{\text {th }}$ generation technology of wireless communication the mobile consumer has given more priority compared to other wireless communication technologies. $5 \mathrm{G}$ technology represents $5^{\text {th }}$ generation mobile communication technology. It is use to make use of mobile within high bandwidth. $5 G$ technology becomes most dominant technology in near future due to presence of additional features such as high data rate, effective billing system, cognitive radio technology, etc. In the last few years wireless technology experienced so many peers of technology from $0 G$ to $4 G$. Presently, the execution work of $5 G$ is going on which concentrates the advance implementation of these technologies. Now, $5 G$ is the term which is not officially used for any existing technology. 5G technology has extraordinary data capabilities and also has capacity to tie together with unrestricted call volumes and high data broadcast within the latest mobile operating system. The router and switch technology used in $5 G$ provides high connectivity. This paper aims at highlighting some of the concept and technologies which will facilitate the affordable provision of very high data rate with the universal coverage and some of its features along with the benefits in $5 G$ wireless networks.
\end{abstract}

Keywords: WLAN, 5G, WMAN, WWWW.

\section{INTRODUCTION}

Mobile and wireless network have made incredible development in the last few years. At the present time many mobiles have also a WLAN adapter. We are using the IP for both generations $2.5 \mathrm{G}$ and $3 \mathrm{G}$, raised study on their integration. Concerning the $4 \mathrm{G}$, its focus is towards flawless incorporation of cellular network such as GSM,3G. Multimode consumers are seen for $4 \mathrm{G}$ but security mechanisms in wireless technology remains a test. There is no merging of different wireless access technology for the same session. The $5 \mathrm{G}$ terminals will have software define radios, modulation scheme and new error control methodologies. The consumer terminals are get focused on $5 \mathrm{G}$ mobile networks which must be capable to merge the special flow from different technologies. The $5 \mathrm{G}$ terminal will make the ultimate selection among different mobile access network providers for a specified service. This thesis gives the concept of intelligent internet phone where, the mobile can prefer the finest connection.

\section{CHALLENGES IN MIGRATION FROM 4G}

\subsection{Multi Mode User Terminal}

The necessity of $4 \mathrm{G}$ technology is to plan a single user station that can be operated in different wireless network. Which can be solved by using cognitive radio technology approach in $5 \mathrm{G}$

\subsection{Choice among Various Wireless System}

Each wireless system has its different characteristics and roles. The choice of most appropriate technology at specific time and place is made according to the consumer quality of service requirement.

\subsection{Security}

Reconfigurable, adaptive and lightweight protection mechanism should be design.

\subsection{Network Infrastructure and QOS Support}

QOS assurance for end to end services that engages systems and IP and non IP based system is challenge.

\subsection{Charging and Billing}

It is hard to handle the accounts of consumers of various service providers. In this way the billing is also difficult task.

\subsection{Data Encryption}

If the GPS receiver will communicate with main transmitter then it is not tough to break the communication link between them. so the consumer must use the encrypted data. 


\section{KEY TERMS OF 5G TECHNOLOGY}

1) $5 \mathrm{G}$ is the completed wireless communication with no limitation.

2) Additional features such as multimedia newspapers, also to watch television with clarity.

3) We can send the data much faster than that of previous generations.

4) 5G will bring World Wide Wireless Web (WWWW).

5) Real wireless world with no more limitation to access and zone issue.

6) Internet protocol version (IPv6) is used in 5G, where a IP address is allotted according to the connection network.

7) The user can simultaneously connected to several wireless access technologies such as zigbee, WiFi, Bluetooth ,etc and seamlessly move between them.

8) Cognitive radio technology, i.e. smart radio permitting unlike radio technologies to the same frequency band efficiently.

9) High altitude stratospheric platform station systems.

\section{STRUCTURE OF 5G}

\begin{tabular}{|l|l|}
\hline Application layer & $\begin{array}{l}\text { Application } \\
\text { (services) }\end{array}$ \\
\hline Presentation layer & Open transport protocol \\
\hline Session layer & \\
\hline Transport layer & Upper network layer \\
\cline { 2 - 2 } Network layer & Lower network layer \\
\hline Data link layer & $\begin{array}{l}\text { Open wireless } \\
\text { architecture }\end{array}$ \\
\hline Physical layer & stack for 5 \\
\hline
\end{tabular}

Fig.1 Protocol stack for $5 \mathrm{G}$

\subsection{Physical layer/ MAC Layer}

For these two layers the $5 \mathrm{G}$ mobile network is expected to be created upon the wireless design.

\subsection{Network Layer}

The network layer has limited address space and no real probability for quality of service support per flow are the main problems present here. These concerns are resolved in IPv6, but traded with significantly bigger header packet. All mobile network will use mobile IP in $5^{\text {th }}$ generation and each mobile station will be foreign agent. However, the mobile can be attached to several mobile phones or wireless networks at the same time.

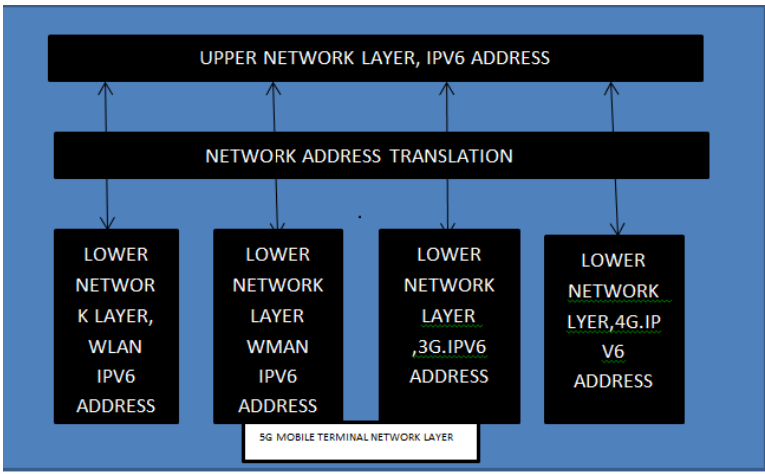

Fig.2 5G mobile terminal network

In this case it will maintain the different internet protocol addresses for each of the radio interference. The $5 \mathrm{G}$ mobile shall maintain virtual multiwireless network environment. For that there must be separation of this layer in two parts in sub-layers in 5G mobiles. i.e. lower network for each interface and upper network for each mobile terminal. The mediator between the upper and lower network layer shall maintain the address translation from upper network layer to lower network layer and vice-versa.

\subsection{Open Transport Protocol Layer}

Transport layer differ the mobile and wireless network from wired network. In all TCP versions the postulation is that the lost segments are due to the network congestion, while in wireless network losses may occur due to greater bit error ratio. Therefore, TCP adaptations and modifications are proposed for the mobile which retransmit the lost or damaged TCP over the wireless link. For 5G mobile terminals downloadable and installed possible transport layer will be suitable.

\subsection{Application Layer}

In case of applications, the final request from the $5 \mathrm{G}$ mobile is to provide the intelligent QOS management over a variety of network. The $5 \mathrm{G}$ phones offer the opportunity for service quality testing and storage of information in information data base in mobile terminal. In the upcoming wireless networks there must be a low complication of execution and an efficient means of concession between the end user and wireless infrastructure.

\section{Features:}

1) 5G technology offers high resolution for bidirectional large bandwidth shaping.

2) Billing system of $5 \mathrm{G}$ technology is more effective and attractive.

3) For fast action $5 \mathrm{G}$ technology provides subscriber supervision tool.

4) Policy to avoid the error is used in $5 \mathrm{G}$ for high quality service.

5) 5G provides large broadcasting of data in terms of gigabit.

6) 5G technology becomes more accurate because of traffic statistics.

7) The remote diagnostic is great feature of $5 \mathrm{G}$. 
8) $5 \mathrm{G}$ provides up to $25 \mathrm{Mbps}$ connectivity speed.

9) $5 \mathrm{G}$ supports virtual private network.

10) Uploading and downloading speed of $5 \mathrm{G}$ is touching peak.

\section{5G ARCHITECTURE}

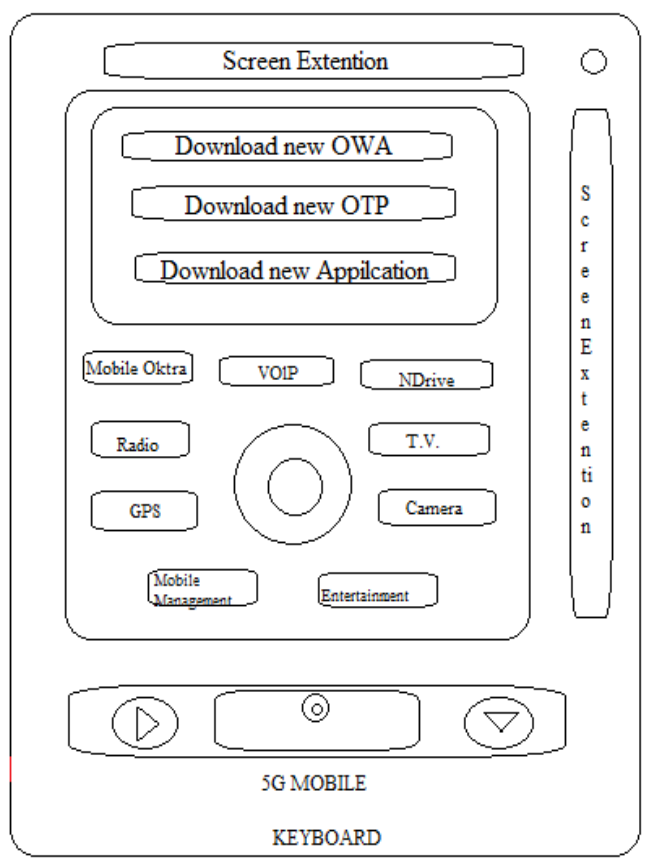

Fig.3 5G mobile design

$5 \mathrm{G}$ is developed to accommodate the QOS and rate necessities set by upcoming applications like wireless broadband access, mobile T. V., multimedia messaging services, video chat, digital video broadcasting (DVB) and other services that utilize bandwidth. The $5 \mathrm{G}$ is used to provide acceptable RF coverage, to interconnect all wireless networks to provide seamless experience to users.

\section{VISIONS AND REQUIREMENTS FOR 5G}

\section{NETWORK}

5G mobile and wireless communication necessitates a mix of new system concept which contains the spectral and energy efficiency. The requirements and visions are outlined below.

\subsection{Data Rate and Latency}

For condensed metropolitan areas, 5G networks are suggested to enable in $95 \%$ of location and time an experience data rate of $300 \mathrm{Mbps}, 60 \mathrm{Mbps}$ in downlink and uplink, respectively. The detailed requirements for different circumstances are listed.

\subsection{Machine Type Communication Devices}

MTC devices are outnumbered the number of traditional human centric devices with internet connectivity. Which may be utilized in vehicles, home appliances and sensors.

\subsection{Millimetre Wave Communication}

To satisfy requirement of increase in traffic and addition of different services, additional frequency band beyond what was previously assigned to $4 \mathrm{G}$ standard is required for. The use of millimeter wave frequency bands is necessary to overcome the problem of rare spectrum resources since it permits transmission at wider band widths than conventional $20 \mathrm{MHz}$ channel for $4 \mathrm{G}$ system.

\subsection{Multiple RATs}

$5 \mathrm{G}$ is not about substituting the existing technology but, it is about augmenting and supporting them with new technology. In 5G systems the GSM (Global System for Mobile communication) and LTE will continue to develop and provide a system with more performance. They were also accompanied by other technologies.

\subsection{Base Station Densification}

BS densification is an effective technology to meet the requirements of $5 \mathrm{G}$ networks. Specifically in $5 \mathrm{G}$ network there will be distributions of a large number of low power nodes, device to device communication links with much higher density than today's macrocell network. Following figure shows multitier network within a macrocell overlaid by relays picocells, device to device communication (D2D) links. The implementation of multiple tiers in cellular network will result in better performance provided that the inter-tier and intra-tier interferences are managed.

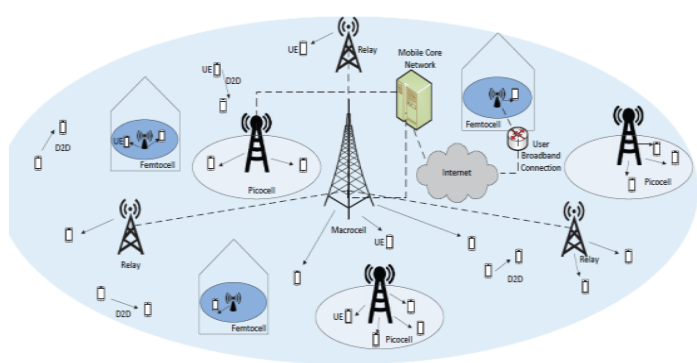

Fig. 4 5G Network with different devices [3]

\subsection{Prioritised Spectrum Access}

The concepts of both traffic and tier based significances will based on 5G network. Traffic based priority arises from different requirements of users. Whereas, the tier based priority is based on different network tier. In the uplink network direction the macrocell user at the cell superiority usually transmit with high powers which produces with high uplink interference to nearby femtocells.

\subsection{Network Assisted Device to Device [D2D]}

\section{Communication}

In the LTE Rel-12 focus will be on network controlled device to device communication where the macrocell base station performs control signalling in terms of synchronization signal configuration, providing uniqueness and safety management. This feature will utilized in $5 \mathrm{G}$ 
network to allow other nodes instead of macrocell base station, to have control.

\section{KEY CHALLENGES}

Assimilation of various standards: Every engineering practice has their own standards, assimilation of these various standards necessitates organized and time consuming approach.

\section{CONCLUSIONS AND FUTURE SCOPE}

Here, in conclusion of $5 \mathrm{G}$ mobile communication survey, the 5G technology is designed as an open platforms on different layers from physical to application. Now the current work is in the module which offers the best operating system and low cost for service. There are lots of improvements from generation 1 to generation 5 in the world of wireless communication technology. This upcoming technology is available in the market at inexpensive cost, high peak expectations than previous one. $5 \mathrm{G}$ technology offers high resolution for avid mobile phone consumers.

\section{ACKNOWLEDGEMENTS}

I wish to express my sincere thanks and deep sense of gratitude to respected guide Prof. D M. Bhalerao in Department of Electronics and Telecommunication Engineering of Sinhgad college of Engineering, Vadgaon (BK), Pune 41, for the technical advice, encouragement and constructive criticism, which motivated to strive harder for excellence.

\section{REFERENCES}

[1] Ms.Sachi Pande ,Manoj Kumar,Ptendra Panwar,Ishita singh"A Survey wireless technology generations with 5G",IJERT Vol.2,Issue 4 April2013.

[2] M.Hata,"Fourth generation mobile communication systems beyond IMT 2000 communications"Proc. $5^{\text {th }}$ asia pacific conf.vol.1 1999 pp.76567

[3] Akhilesh kumar pachauri, Ompal singh"5G Technology -redifining wireless communication in upcoming years" IJCSE vol.1 issue 1 August 2012.

[4] Ramnarayan, Vashu kumar, Vipin kumar "A New generation mobile network 5G'IJCA Vol.17 no.20 May 2013.

[5] E.Hossain , M.Rasti, H. Tabassum, A.Abdelnaser "Evolution toward 5G multitier cellular wireless network", Xiv:1401.553v1[cs.N1]22Jan 2012

[6] Toni janewaski, "Traffic analysis and design of wireless IP network"Artech house inc.Boston USA May 2003

[7] Janie McNair, Fang zhu,"Vertical handoffs in fourth generation multinetwork encironment" IEEE Wireless communication June 2004

[8] Jivesh govil, jivika govil,"5G Functionallity development and an analysis of mobile wireless grid"first international conference on emerging trends in engineering and technology.
[9] Toni jaweski "A system for PLMN WLAN internet working"journal communication and networks pp 192.vol.7, No. 2, June 2005

[10] A.Bria,F.Gesseler, O.Queseth, R.Sridth,M.Unbehaun" $4^{\text {th }}$ generation wireless infrastructure scenario and research challenges" IEEE personal communication vol. 8, No. 6, December 2001

[11] M.Bhalla, A. Bhalla,"Generations of mobile wireless technology : a survey"international journal of computer application Vol. 5, No. 4 August 2010

[12] T . Janweski "5G Mobile phone concept"CCNC Conference in LAS vegas, 2009

[13] T .Tudzaw T. Janewaski "Design of 5G mobile architechture" international journal of communication networks and information security,vol.3 ,NO. 2, August 2011 